\title{
Coordinación entre servicios farmacéuticos para una farmacoterapia integrada: el caso de Cataluña
}

\author{
Coordination between pharmaceutical services \\ for integrated pharmacotherapy: the case of Catalonia
}

Karen Sarmento Costa ${ }^{1}$ Moisés Goldbaum ${ }^{1}$ Rafel Guayta-Escolies ${ }^{2}$ Pilar Modamio ${ }^{3}$ Eduardo Luis Mariño ${ }^{3}$ José Luis Segú Tolsá ${ }^{3}$
${ }^{1}$ Departamento de Medicina Preventiva, Faculdade de Medicina, Universidade de São Paulo. Av. Dr. Arnaldo 455, Cerqueira César. 01246903 São Paulo SP Brasil.

karen.costa@gmail.com

${ }^{2}$ Consell de Collegis

Farmacèutics de Catalunya.

Barcelona Catalunya

España.

${ }^{3}$ Unidad de Farmacia

Clínica y Farmacoterapia,

Departamento de Farmacia

y Tecnología Farmacéutica,

Facultad de Farmacia y

Ciencias de la Alimentación,

Universidad de Barcelona.

Barcelona Catalunya

España.
Abstract Pharmaceutical policies have been considered strategies to contribute to the guarantee of care coordination and clinical integration. This study sought to describe the pharmaceutical services developed at different levels of care in the health network in Catalonia, as well as to identify and analyze the mechanisms and instruments that act as facilitators and/or barriers to the coordination of pharmacotherapy. This is a descriptive study of 12 cases of hospital pharmacy services, primary care and community pharmacies. Advances related to the perception, formalization and clinical and assistance coordination of the pharmaceutical services were identified. However, weaknesses and potential improvements in coordination were observed. The conclusion drawn was that the different tools and instruments implemented appear to facilitate a greater possibility of integration between pharmaceutical services and the latter with the health services network to contribute to integrated pharmacotherapy.

Key words Health systems, Pharmaceutical policies, Pharmaceutical services, Care coordination
Resumen Las politicas farmacéuticas han sido consideradas como estratégicas para contribuir con la garantía de la coordinación asistencial y la integración clínica. El presente estudio tiene como objetivo describir los servicios farmacéuticos desarrollados en diferentes niveles asistenciales en la red de salud de Cataluña, así como identificar y analizar los mecanismos e instrumentos que actúan como facilitadores y/o barreras para la coordinación de la farmacoterapia. Se trata de un estudio descriptivo de 12 casos de los servicios farmacéuticos hospitalarios, atención primaria y oficinas de farmacia comunitarias. Se identifica avances que relacionan la percepción, formalización y la coordinación asistencial y clínica de los servicios farmacéuticos. Sin embargo, se identifican también fragilidades y situaciones mejorables en cuanto a la coordinación. Se concluyó que las diferentes herramientas $e$ instrumentos implantados, parece facilitar una mayor posibilidad de integración entre servicios farmacéuticos y de éstos con la red de salud para contribuir con una farmacoterapia integrada.

Palabras clave Sistemas de salud, Politicas farmacéuticas, Servicios farmacéuticos, Coordinación asistencial 


\section{Introducción}

Los sistemas universales de salud se enfrentan a un conjunto de desafíos para garantizar la implementación de sus directrices y posterior consolidación. Entre éstos, la garantía de la coordinación asistencial y la integración clínica han sido consideradas como objetivo prioritario en muchos de ellos, con la intención de mejorar la calidad y la eficiencia en la atención a problemas crónicos, que requieren intervención de diversos profesionales y servicios ${ }^{1}$.

La coordinación puede entenderse como la capacidad de los prestadores de servicios de coordinar el uso de estos servicios entre niveles de atención, para resolver necesidades menos frecuentes y más complejas, y así garantizar la continuidad asistencial ${ }^{2}$. Kringos et al. ${ }^{3}$ en la revisión sistemática sobre los cuidados primarios en la salud, apunta que una coordinación asistencial aumenta la calidad en la atención y reduce costes $^{3}$. Además, puede facilitar un seguimiento adecuado, minimiza el riesgo de cometer errores, previene complicaciones y mejora la seguridad de los pacientes ${ }^{4,5}$. En las últimas décadas se han desarrollado diferentes intervenciones dirigidas a mejorar estos aspectos, tanto a nivel político (planes intersectoriales, sistemas de compra y financiación) como organizativo (organizaciones integradas, introducción de mecanismos y estrategias de coordinación $)^{1}$.

En la Unión Europea, desde la década de 1990, se están implementando reformas cuyo objetivo es la mejora de la coordinación, sobre todo dirigidas al fortalecimiento del primer nivel de atención ${ }^{6,7}$. Por otro lado, en la región de América Latina todavía son incipientes las reformas centradas en la coordinación de los cuidados, lo cual se refleja en el reducido número de estudios que tratan de este tema ${ }^{5}$.

Entre las políticas públicas sectoriales y transversales a los sistemas de salud, las políticas farmacéuticas han sido consideradas como estrategias fundamentales para contribuir en el proceso de coordinación asistencial e integración clínica, en especial en sistemas integrados a partir de las perspectivas de redes de salud.

Es necesario que el paciente se vea de forma transversal en los Sistemas de Salud, y para eso, el medicamento debe tener su gestión integrada. Por tanto, un nivel de coordinación de decisiones y cuidados farmacoterapéuticos, capaces de integrar coherentemente las acciones de diferentes profesionales para garantizar la máxima efectividad y el mínimo riesgo en las terapias, se constituye en el factor facilitador del continuo asistencial. Desde esta perspectiva, en el proceso de coordinación asistencial y de integración clínica, los servicios farmacéuticos pueden y deben actuar como catalizadores de todos los elementos de apoyo a las actividades y criterios clínicos entre profesionales, y también entre profesionales y pacientes 8 .

La Organización Panamericana de la Salud considera que los servicios farmacéuticos deben orientase hacia la eliminación de las dificultades de acceso; la mejoría de resultados terapéuticos y de salud; la incorporación de los servicios farmacéuticos como componentes de las políticas farmacéuticas nacionales; la gestión con atención integral e integrada, comprometida con el logro de resultados en salud; y la prestación de los servicios farmacéuticos basados en el individuo, la familia y la comunidad?.

El presente artículo tiene como objetivo describir los servicios farmacéuticos desarrollados en diferentes niveles asistenciales en la red de salud de Cataluña (Comunidad Autónoma del estado Español), así como identificar y analizar los mecanismos e instrumentos que actúan como facilitadores y/o barreras para la coordinación de la farmacoterapia en el Sistema de Salud.

\section{Método}

Estudio de caso, que sigue lo establecido en la metodología de la investigación Equity-LA ${ }^{2,10}$. El estudio fue desarrollado en la Comunidad $\mathrm{Au}$ tónoma de Cataluña, en España, que tiene una población de más de 7,5 millones de personas. Fueron definidas las ciudades de Barcelona (1,6 millones de habitantes), Mataró (124.280 millones de habitantes), Terrasa (215.517 millones de habitantes), San Cugat del Vallés (87.118 millones de habitantes) que ofrecen servicios farmacéuticos en todos los niveles Sistema de Salud.

La muestra fue definida por conveniencia y los criterios de inclusión fueron: los servicios farmacéuticos oficialmente establecidos en el Sistema de Salud de Cataluña, que atendían a los diferentes ámbitos asistenciales y respondían a los objetivos de la investigación, así como por la aceptación de las organizaciones en participar de la misma.

Se constituyeron como participantes de la encuesta, personas clave que podrían opinar sobre el tema de los servicios farmacéuticos prestados en el entorno. Los profesionales fueron seleccionados en las unidades prestadoras de los servicios 
de salud, por los siguientes criterios: experiencia de más de 5 años en el área, responsable por la gestión de la unidad de prestación de servicios y/o de los servicios farmacéuticos y cubrir los distintos niveles de atención.

Fue enviada una carta a cada gestor de las organizaciones de salud explicando los objetivos y otros datos de la investigación. Fue solicitada la autorización para llevar a cabo la encuesta y fueron invitados a participar de acuerdo con la disponibilidad y los criterios de inclusión.

En primer lugar, las Organizaciones Sanitarias Integradas $(n=3)$, son redes de servicios de salud que ofrecen una atención coordinada a una población determinada a través de prestaciones de servicios de salud. Se responsabilizan de los costes y resultados de la salud de la población, abarcando la atención primaria, la atención especializada y la atención sociosanitaria, así como los tratamientos de rehabilitación.

Se cuenta con 12 casos de estudios que integran los tres tipos de servicios farmacéuticos del sistema local: servicios farmacéuticos hospitalarios $(\mathrm{SFH})$, servicios farmacéuticos de atención primaria (SFAP) y servicios farmacéuticos de las farmacias comunitarias (SFFC).

Los servicios farmacéuticos analizados estaban integrados en diferentes organizaciones prestadoras. En primer lugar, las Organizaciones Sanitarias Integradas $(\mathrm{n}=3)$, que son redes de servicios de salud que ofrecen una atención coordinada a una población determinada, a través de prestaciones de servicios de salud, y se responsabiliza de los costes y resultados en salud de la población ${ }^{2}$ y abarca la atención primaria, la atención especializada y la atención sociosanitaria, así como los tratamientos de rehabilitación. En segundo lugar, los Centros de Atención Primaria $(\mathrm{n}=3)$, punto principal de acceso al sistema de salud, en el cual se ofrecen servicios de un equipo multiprofesional en el ámbito de la atención primaria. En tercer lugar, los Centros Hospitalarios $(\mathrm{n}=2)$, punto de acceso a la atención especializada, en el cual se ofrecen servicios para condiciones agudas y crónicas. Finalmente, las Farmacias Comunitarias $(\mathrm{n}=4)$, establecimientos privados estructurados que tienen como objetivo garantizar una correcta distribución y fácil acceso a los medicamentos por parte de los ciudadanos y que están contratadas por el SNS español para dispensar los medicamentos. Se diseñó un cuestionario estructurado y organizado en tres bloques temáticos: caracterización general de las organizaciones/establecimientos de salud, caracterización general de los servicios farmacéuticos y coordinación clínica, que contenía 92 preguntas, abiertas y/o cerradas. Para el presente estudio, se hizo un corte de las cuestiones relacionadas con los servicios farmacéuticos clínicos y la coordinación clínica.

Las entrevistas se realizaron individualmente con los farmacéuticos responsables por los servicios farmacéuticos asistenciales y personas del ámbito gerencial de las organizaciones. Sin embargo, este estudio utilizó sólo el instrumento de profesionales farmacéuticos.

Las entrevistas se llevaron a cabo entre los meses de enero a mayo de 2016. El cuestionario se desarrolló sobre el Software Epi Info versión 7 y se realizaron de forma presencial con el apoyo de un computador para el registro de los datos.

\section{Resultados}

El Cuadro 1 presenta la caracterización general de los casos analizados, en cuanto a su clasificación, titularidad, financiación y clientes. Son cuatro los tipos de clasificación de las organizaciones: organizaciones sanitarias integradas $(\mathrm{n}=3)$ todas ellas de titularidad pública y financiación pública exclusivas; centros de atención primaria/sector sanitario de atención primaria $(\mathrm{n}=3)$, siendo dos de titularidad pública con financiación pública exclusiva y uno privado con financiación mixta; Centros hospitalarios $(\mathrm{n}=2)$, siendo uno de titularidad privada lucrativa y financiación mixta y otro de titularidad pública con financiación pública; y farmacias comunitarias $(\mathrm{n}=4)$, privadas con fines lucrativos y financiación mixta.

Los clientes internos para los servicios farmacéuticos hospitalarios y de atención primaria son prioritariamente los médicos, enfermeras y la dirección de la organización; y en las farmacias comunitarias son médicos y enfermeros del SNS y farmacéuticos de otros servicios asistenciales. Para los tres tipos de servicios farmacéuticos los clientes externos son prioritariamente los pacientes.

El Cuadro 2 presenta la caracterización de los servicios farmacéuticos analizados en cuanto a su misión, servicios prestados y cartera de servicios farmacéuticos clínicos. En relación a la misión de los servicios farmacéuticos, se ha observado que los entrevistados indicaron que, en general, son servicios dirigidos a garantizar el acceso y uso racional a los medicamentos por parte de la población, queriendo contribuir a los resultados terapéuticos en salud de los individuos y de la población de responsabilidad. 
Cuadro 1. Caracterización general de los casos analizados. Cataluña, 2016.

\begin{tabular}{|c|c|c|c|c|c|}
\hline Casos & $\begin{array}{c}\text { Clasificación de la } \\
\text { organización }\end{array}$ & Titularidad & Financiación & Clientes Internos & Clientes Externos \\
\hline 1 & $\begin{array}{l}\text { Organización } \\
\text { Sanitaria Integrada }\end{array}$ & Pública & $\begin{array}{l}\text { Público } \\
\text { exclusivo }\end{array}$ & $\begin{array}{l}\text { Médicos, enfermeros, } \\
\text { dirección de la } \\
\text { organización }\end{array}$ & Pacientes \\
\hline 2 & $\begin{array}{l}\text { Organización } \\
\text { Sanitaria Integrada }\end{array}$ & Pública & $\begin{array}{l}\text { Público } \\
\text { exclusivo }\end{array}$ & $\begin{array}{l}\text { Médicos, enfermeros, } \\
\text { dirección de la } \\
\text { organización }\end{array}$ & Pacientes \\
\hline 3 & $\begin{array}{l}\text { Organización } \\
\text { Sanitaria Integrada }\end{array}$ & Pública & $\begin{array}{l}\text { Público } \\
\text { exclusivo }\end{array}$ & $\begin{array}{l}\text { Médicos, enfermeros, } \\
\text { dirección de la } \\
\text { organización }\end{array}$ & Pacientes \\
\hline 4 & $\begin{array}{l}\text { Sector sanitario de } \\
\text { atención primaria }\end{array}$ & Pública & $\begin{array}{l}\text { Público } \\
\text { exclusivo }\end{array}$ & $\begin{array}{l}\text { Médicos y enfermeros } \\
\text { del equipo de Atención } \\
\text { Primaria }\end{array}$ & Pacientes \\
\hline 5 & $\begin{array}{l}\text { Centro de Atención } \\
\text { Primaria }\end{array}$ & Pública & Mixto & $\begin{array}{l}\text { Médicos, enfermeros, } \\
\text { dirección de la } \\
\text { organización }\end{array}$ & Pacientes \\
\hline 6 & $\begin{array}{l}\text { Centro de Atención } \\
\text { Primaria }\end{array}$ & Privada & $\begin{array}{l}\text { Público } \\
\text { exclusivo }\end{array}$ & $\begin{array}{l}\text { Médicos, enfermeras y } \\
\text { administrativos }\end{array}$ & Pacientes \\
\hline 7 & Hospital & $\begin{array}{l}\text { Privada } \\
\text { con fines } \\
\text { lucrativos }\end{array}$ & Misto & $\begin{array}{l}\text { Dirección, servicios } \\
\text { médicos y enfermería }\end{array}$ & $\begin{array}{l}\text { Pacientes, aseguradoras } \\
\text { Organización } \\
\text { corporativa }\end{array}$ \\
\hline 8 & Hospital & $\begin{array}{l}\text { Privada } \\
\text { sin fines } \\
\text { lucrativos }\end{array}$ & Misto & $\begin{array}{l}\text { Pacientes, médicos, } \\
\text { enfermeras, dirección del } \\
\text { hospital }\end{array}$ & Pacientes \\
\hline \multirow[t]{2}{*}{9} & \multirow[t]{2}{*}{$\begin{array}{l}\text { Farmacia } \\
\text { Comunitaria }\end{array}$} & $\begin{array}{l}\text { Privado } \\
\text { con fines } \\
\text { lucrativos }\end{array}$ & Mixto & \multirow{2}{*}{$\begin{array}{l}\text { Médico y enfermero (en } \\
\text { el SNS) y farmacéuticos } \\
\text { de otros servicios } \\
\text { asistenciales }\end{array}$} & Pacientes \\
\hline & & $\begin{array}{l}\text { Privado } \\
\text { con fines } \\
\text { lucrativos }\end{array}$ & Mixto & & $\begin{array}{l}\text { Logística (distribuidores } \\
\text { y proveedores) } \\
\text { demás servicios } \\
\text { farmacéuticos }\end{array}$ \\
\hline 10 & $\begin{array}{l}\text { Farmacia } \\
\text { Comunitaria }\end{array}$ & $\begin{array}{l}\text { Privado } \\
\text { con fines } \\
\text { lucrativos }\end{array}$ & Mixto & $\begin{array}{l}\text { Médico y enfermero (en } \\
\text { el SNS) y farmacéuticos } \\
\text { de otros servicios } \\
\text { asistenciales }\end{array}$ & $\begin{array}{l}\text { Pacientes } \\
\text { Logística (distribuidores } \\
\text { y proveedores) } \\
\text { demás servicios } \\
\text { farmacéuticos }\end{array}$ \\
\hline 11 & $\begin{array}{l}\text { Farmacia } \\
\text { Comunitaria }\end{array}$ & $\begin{array}{l}\text { Privado } \\
\text { con fines } \\
\text { lucrativos }\end{array}$ & Mixto & $\begin{array}{l}\text { Médico y enfermero (en } \\
\text { el SNS) y farmacéuticos } \\
\text { de otros servicios } \\
\text { asistenciales }\end{array}$ & $\begin{array}{l}\text { Pacientes } \\
\text { Logística (distribuidores } \\
\text { y proveedores) } \\
\text { y demás servicios } \\
\text { farmacéuticos }\end{array}$ \\
\hline 12 & $\begin{array}{l}\text { Farmacia } \\
\text { Comunitaria }\end{array}$ & $\begin{array}{l}\text { Privado } \\
\text { con fines } \\
\text { lucrativos }\end{array}$ & Mixto & $\begin{array}{l}\text { Médico y enfermera (en } \\
\text { el SNS) y farmacéuticos } \\
\text { de otros servicios } \\
\text { asistenciales }\end{array}$ & $\begin{array}{l}\text { Pacientes } \\
\text { Logística (distribuidores } \\
\text { y proveedores) y demás } \\
\text { servicios farmacéuticos }\end{array}$ \\
\hline
\end{tabular}

Se verificó la existencia de una cartera de servicios farmacéuticos clínicos en los casos analizados. Es común en todos los diferentes servicios la selección, evaluación, recomendación de los medicamentos, validación de la prescripción y acciones de farmacovigilancia. En los servicios farmacéuticos en las OSI, la cartera es más amplia, con acciones de apoyo a las decisiones clínicas de los profesionales, conciliación de los medicamentos, seguimiento, atención farmacéu- 
Cuadro 2. Caracterización de los casos de servicios farmacéuticos analizados. Cataluña, 2016.

\begin{tabular}{|c|c|c|c|}
\hline Casos & $\begin{array}{l}\text { Misión de los servicios } \\
\text { farmacéuticos }\end{array}$ & \begin{tabular}{|c|} 
Servicio Farmacéutico \\
prestado por nivel de atención
\end{tabular} & Cartera de Servicios Farmacéuticos Asistencial \\
\hline 1 & $\begin{array}{l}\text { Proporcionar servicios a los } \\
\text { clientes externos e internos para } \\
\text { contribuir a la mejora de la calidad } \\
\text { asistencial mediante una asistencia } \\
\text { individualizada con seguridad, } \\
\text { efectividad a la farmacoterapia y } \\
\text { eficiencia }\end{array}$ & $\begin{array}{l}\text { Servicio Farmacéutico } \\
\text { Hospitalario presta servicios } \\
\text { para la atención primaria, } \\
\text { hospitalaria y sociosanitaria }\end{array}$ & $\begin{array}{l}\text { Farmacocinética clínica } \\
\text { Apoyo a decisión clínica de los profesionales } \\
\text { médicos y enfermeros } \\
\text { Desarrollo de programas de políticas de } \\
\text { medicamentos de alcance poblacional y/o } \\
\text { territorial } \\
\text { Pacientes de alta: conciliación e información de } \\
\text { los medicamentos } \\
\text { Dispensación de medicamentos, seguimiento } \\
\text { farmacoterapéutico, detección de problemas } \\
\text { relacionados con los medicamentos (PRM), } \\
\text { educación en salud } \\
\text { Atención farmacéutica a pacientes } \\
\text { hospitalizados, pacientes ambulatorios a los } \\
\text { que se dispensan los medicamentos en todos } \\
\text { aquellos ámbitos }\end{array}$ \\
\hline 2 & $\begin{array}{l}\text { Añadir valor a luz de los } \\
\text { medicamentos en la organización, } \\
\text { conseguir mejorar los resultados y la } \\
\text { eficiencia }\end{array}$ & $\begin{array}{l}\text { Servicio Farmacéutico } \\
\text { Hospitalario presta servicios } \\
\text { para la atención primaria, } \\
\text { hospitalaria y sociosanitaria }\end{array}$ & $\begin{array}{l}\text { Pacientes de alta: conciliación e información de } \\
\text { los medicamentos } \\
\text { Apoyo a decisión clínica de los profesionales } \\
\text { médicos y enfermeros } \\
\text { Desarrollo de programas de políticas de } \\
\text { medicamentos de alcance poblacional y/o } \\
\text { territorial } \\
\text { Dispensación de medicamentos, seguimiento } \\
\text { farmacoterapéutico, detección de problemas } \\
\text { relacionados con los medicamentos (PRM), } \\
\text { educación en salud } \\
\text { Atención farmacéutica a pacientes } \\
\text { hospitalizados, pacientes ambulatorios a los } \\
\text { que se dispensan los medicamentos en todos } \\
\text { aquellos ámbitos }\end{array}$ \\
\hline 3 & $\begin{array}{l}\text { Mejorar la gestión de los } \\
\text { medicamentos y contribuir con la } \\
\text { salud del paciente }\end{array}$ & $\begin{array}{l}\text { Servicio farmacéutico de } \\
\text { APS presta servicios para la } \\
\text { atención primaria } \\
\text { Servicio farmacéutico } \\
\text { hospitalario presta servicio a } \\
\text { nivel sociosanitario }\end{array}$ & $\begin{array}{l}\text { Atención farmacéutica a pacientes internados } \\
\text { Apoyo a decisión clínica de los profesionales } \\
\text { médicos y enfermeros } \\
\text { Pacientes de alta: conciliación e información de } \\
\text { los medicamentos } \\
\text { Dispensación de medicamentos, seguimiento } \\
\text { farmacoterapéutico, detección de problemas } \\
\text { relacionados con los medicamentos (PRM), } \\
\text { educación en salud }\end{array}$ \\
\hline 4 & $\begin{array}{l}\text { Promover, dibujar coordinar, } \\
\text { implementar e evaluar las } \\
\text { actividades y mejorar el proceso } \\
\text { de utilización de medicamentos } \\
\text { de prescripción de acuerdo con la } \\
\text { evidencia científica, con criterios de } \\
\text { eficiencia y seguridad, de acuerdo } \\
\text { con la política y estrategia del } \\
\text { Instituto Catalán de la Salud (ICS) } \\
\text { y del Servicio Catalán de la Salud } \\
\text { (CatSalut) a fin de aumentar la } \\
\text { calidad de los cuidados y garantizar } \\
\text { la sostenibilidad en relación con el } \\
\text { uso de los medicamentos }\end{array}$ & \begin{tabular}{|l|} 
Servicio farmacéutico de APS: \\
responsable de la coordinación \\
de los servicios farmacéuticos \\
de la atención primaria
\end{tabular} & $\begin{array}{l}\text { Apoyo a decisión clínica de los profesionales } \\
\text { médicos y enfermeros } \\
\text { Desarrollo de programas de políticas de } \\
\text { medicamentos de alcance poblacional y/o } \\
\text { territorial }\end{array}$ \\
\hline
\end{tabular}


Cuadro 2. continuación

\begin{tabular}{|c|c|c|c|}
\hline Casos & $\begin{array}{c}\text { Misión de los servicios } \\
\text { farmacéuticos }\end{array}$ & \begin{tabular}{|c} 
Servicio Farmacéutico \\
prestado por nivel de atención
\end{tabular} & Cartera de Servicios Farmacéuticos Asistencial \\
\hline 5 & $\begin{array}{l}\text { Atención a las necesidades e } \\
\text { informaciones farmacoterapéuticas } \\
\text { de los profesionales sanitarios en } \\
\text { referencia con su ámbito de sistema } \\
\text { de salud }\end{array}$ & $\begin{array}{l}\text { Servicio farmacéutico de } \\
\text { APS presta servicios para la } \\
\text { atención primaria }\end{array}$ & $\begin{array}{l}\text { Armonización clínica de los profesionales } \\
\text { médicos y enfermeros } \\
\text { Apoyo a decisión clínica de los profesionales } \\
\text { médicos y enfermeros } \\
\text { Acceso al entorno familiar y políticas de } \\
\text { medicamentos de alcance poblacional /o } \\
\text { territorial } \\
\text { Acceso al entorno familiar y políticas de } \\
\text { medicamentos de alcance poblacional /o } \\
\text { territorial } \\
\text { Coordinación de la atención farmacéutica entre } \\
\text { los niveles }\end{array}$ \\
\hline 6 & $\begin{array}{l}\text { Garantir el acceso a los } \\
\text { medicamentos de forma eficiente, } \\
\text { maximizando los beneficios y } \\
\text { minimizando los riesgos }\end{array}$ & $\begin{array}{l}\text { Servicio farmacéutico da } \\
\text { APS presta servicios para la } \\
\text { atención primaria }\end{array}$ & $\begin{array}{l}\text { Armonización clínica } \\
\text { Atención farmacéutica a pacientes ambulatorios } \\
\text { a los que se dispensa los medicamentos y todos } \\
\text { aquellos de su ámbito } \\
\text { Pacientes de alta: conciliación e información de } \\
\text { los medicamentos } \\
\text { Armonización clínica de los profesionales } \\
\text { médicos y enfermeros } \\
\text { Apoyo a decisión clínica de los profesionales } \\
\text { médicos y enfermeros } \\
\text { Seguimiento farmacoterapéutico, detección de } \\
\text { problemas relacionados con los medicamentos } \\
\text { (PRM), educación en salud } \\
\text { Coordinación de la atención farmacéutica entre } \\
\text { los niveles }\end{array}$ \\
\hline 7 & $\begin{array}{l}\text { Proporcionar farmacoterapia } \\
\text { eficiente e segura }\end{array}$ & $\begin{array}{l}\text { Servicio Farmacéutico } \\
\text { Hospitalario presta servicios a } \\
\text { la atención hospitalaria }\end{array}$ & $\begin{array}{l}\text { Armonización clínica } \\
\text { Atención farmacéutica a pacientes internados y } \\
\text { ambulatorios donde se dispensan medicamentos } \\
\text { Pacientes de alta: conciliación e información de } \\
\text { los medicamentos } \\
\text { Farmacocinética clínica } \\
\text { Armonización clínica de los profesionales } \\
\text { médicos y enfermeros } \\
\text { Apoyo a decisión clínica de los profesionales } \\
\text { médicos y enfermeros }\end{array}$ \\
\hline 8 & $\begin{array}{l}\text { Trabajar para la salud de las } \\
\text { personas impulsando el uso seguro } \\
\text { de los medicamentos }\end{array}$ & $\begin{array}{l}\text { Servicio Farmacéutico } \\
\text { Hospitalario presta servicios a } \\
\text { la atención hospitalaria }\end{array}$ & $\begin{array}{l}\text { Armonización clínica } \\
\text { Atención farmacéutica a pacientes } \\
\text { internados, ambulatorios donde se dispensan } \\
\text { medicamentos } \\
\text { Pacientes de alta: conciliación e informáis de los } \\
\text { medicamentos } \\
\text { Farmacocinética clínica } \\
\text { Armonización clínica de los profesionales } \\
\text { médicos y enfermeros } \\
\text { Apoyo a decisión clínica de los profesionales } \\
\text { médicos y enfermeros }\end{array}$ \\
\hline
\end{tabular}

continua

tica a pacientes ambulatoriales, hospitalaria y al alta. En los servicios en la atención primaria se destaca-se el apoyo a los profesionales médicos y enfermeros, acceso al entorno familiar y políticas de medicamentos de alcance poblacional y/o te- rritorial y la coordinación de la atención farmacéutica entre los niveles.

Las farmacias comunitarias que participaron en el estudio son todas urbanas, en concreto de la ciudad de Barcelona. La cartera de servicios desa- 
Cuadro 2. continuación

\begin{tabular}{|c|c|c|c|}
\hline Casos & $\begin{array}{l}\text { Misión de los servicios } \\
\text { farmacéuticos }\end{array}$ & $\begin{array}{c}\text { Servicio Farmacéutico } \\
\text { prestado por nivel de atención }\end{array}$ & Cartera de Servicios Farmacéuticos Asistencial \\
\hline 9 & $\begin{array}{l}\text { Acercar el medicamento al } \\
\text { ciudadano para que este haga un } \\
\text { uso racional y saludable del mismo }\end{array}$ & $\begin{array}{l}\text { Farmacia Comunitaria: } \\
\text { Presta servicios a pacientes } \\
\text { ambulatorios encaminados } \\
\text { por el SNS y para la población } \\
\text { general }\end{array}$ & $\begin{array}{l}\text { Dispensación de la medicación } \\
\text { Atención farmacéutica: dispensación activa, } \\
\text { Sistema Personalizado de Dosificación -SPD, } \\
\text { seguimiento farmacoterapéutio, educación } \\
\text { e información, identificación de problemas } \\
\text { relacionados con los medicamentos (PRM) } \\
\text { Participación y ejecución de programas de } \\
\text { salud pública: programas de cribado, educación } \\
\text { y promoción de la salud } \\
\text { Prestación de servicios específicos contratados, } \\
\text { como intercambio de jeringas } \\
\text { Acciones en entornos comunitarios y familiares }\end{array}$ \\
\hline 10 & $\begin{array}{l}\text { Mejorar la adherencia a los } \\
\text { tratamientos por parte de los } \\
\text { pacientes }\end{array}$ & $\begin{array}{l}\text { Farmacia Comunitaria: } \\
\text { Presta servicios a pacientes } \\
\text { ambulatorios encaminados } \\
\text { por el SNS y para la población } \\
\text { general }\end{array}$ & $\begin{array}{l}\text { Dispensación de la medicación } \\
\text { Atención farmacéutica: dispensación activa, } \\
\text { Sistema Personalizado de Dosificación -SPD, } \\
\text { identificación de problemas relacionados con } \\
\text { los medicamentos (PRM) } \\
\text { Participación y ejecución de programas de } \\
\text { salud pública: programas de cribado, educación } \\
\text { y promoción de la salud }\end{array}$ \\
\hline 11 & $\begin{array}{l}\text { La protección y mejora de la salud } \\
\text { de sus pacientes/clientes }\end{array}$ & $\begin{array}{l}\text { Farmacia Comunitaria: } \\
\text { Presta servicios a pacientes } \\
\text { ambulatorios encaminados } \\
\text { por el SNS y para la población } \\
\text { general }\end{array}$ & $\begin{array}{l}\text { Dispensación de la medicación } \\
\text { Atención farmacéutica: dispensación activa, } \\
\text { Sistema Personalizado de Dosificación -SPD, } \\
\text { seguimiento farmacoterapéutico, educación } \\
\text { e información, identificación de problemas } \\
\text { relacionados con los medicamentos (PRM) } \\
\text { Participación y ejecución de programas de } \\
\text { salud pública: programas de cribado, educación } \\
\text { y promoción de la salud }\end{array}$ \\
\hline 12 & $\begin{array}{l}\text { Agente de salud de fácil acceso a la } \\
\text { población }\end{array}$ & $\begin{array}{l}\text { Farmacia Comunitaria: } \\
\text { Presta servicios a pacientes } \\
\text { ambulatorios encaminados } \\
\text { por el SNS y para la población } \\
\text { general }\end{array}$ & $\begin{array}{l}\text { Dispensación de la medicación } \\
\text { Atención farmacéutica: dispensación activa, } \\
\text { Sistema Personalizado de Dosificación -SPD, } \\
\text { seguimiento farmacoterapéutico, educación } \\
\text { e información, identificación de problemas } \\
\text { relacionados con los medicamentos (PRM) } \\
\text { Conciliación de la medicación } \\
\text { Acciones en entornos comunitarios y familiares } \\
\text { Participación y ejecución de programas de } \\
\text { salud pública: programas de cribado, educación } \\
\text { y promoción de la salud }\end{array}$ \\
\hline
\end{tabular}

rrollada en las farmacias comunitarias, cuentan para todos los casos con la dispensación de los medicamentos subsidiados por el SNS y actividades vinculadas a la prestación de la atención farmacéutica a los pacientes. También se verificó la dispensación de los medicamentos mediante los sistemas personalizados de dosificación (SPD), la participación y ejecución de programas de selección y la prestación de servicios contratados, por ejemplo, el intercambio de jeringas.
El Cuadro 3 muestra los factores relacionados a la coordinación farmacoterapéutica de la red de servicios de salud y la contribución de los servicios farmacéuticos en los casos concernientes a los servicios farmacéuticos hospitalarios y de atención primaria.

En general los servicios farmacéuticos analizados, en los diferentes tipos de organizaciones colaboran en las actividades destinadas al apoyo clínico de los clientes internos, como la presta- 
Cuadro 3. Los factores relacionados con la coordinación farmacoterapia de la red de servicios de salud y la contribución de los servicios farmacéuticos hospitalarios y de la atención primaria en los diferentes niveles de atención del Sistema de Salud Catalán. Cataluña, 2016.

\begin{tabular}{|c|c|c|c|c|}
\hline Casos & $\begin{array}{c}\text { Contribución de los servicios } \\
\text { farmacéuticos entre los niveles de } \\
\text { atención }\end{array}$ & $\begin{array}{l}\text { Instrumentos y/o mecanismos de } \\
\text { coordinación asistencial }\end{array}$ & $\begin{array}{l}\text { Procesos } \\
\text { asistenciales } \\
\text { priorizados }\end{array}$ & $\begin{array}{c}\text { Profesionales que } \\
\text { participan en el proceso } \\
\text { asistencial }\end{array}$ \\
\hline 1 & $\begin{array}{l}\text { Información sobre la prescripción, } \\
\text { medicación y seguimiento } \\
\text { Participación en la elaboración de } \\
\text { líneas de cuidados y protocolos } \\
\text { asistenciales } \\
\text { Intercambio de conocimiento }\end{array}$ & $\begin{array}{l}\text { Guías terapéuticas, rutas } \\
\text { asistenciales y protocolos } \\
\text { asistenciales } \\
\text { Reuniones periódicas entre los } \\
\text { distintos profesionales de salud, } \\
\text { Comisiones y comités } \\
\text { Objetivos del Servicio Catalán de la } \\
\text { Salud (CatSalut) }\end{array}$ & $\begin{array}{l}\text { Procesos } \\
\text { crónicos, } \\
\text { polimedicados }\end{array}$ & $\begin{array}{l}\text { Médico, enfermero y } \\
\text { farmacéutico }\end{array}$ \\
\hline 2 & $\begin{array}{l}\text { Guía farmacoterapéutica } \\
\text { Comisión de Farmacia integral } \\
\text { Participación en las comisiones } \\
\text { asistenciales }\end{array}$ & $\begin{array}{l}\text { Historia clínica compartida, } \\
\text { Comisión de Farmacia y } \\
\text { Terapéutica } \\
\text { Protocolos } \\
\text { Comité de dirección asistencial } \\
\text { (participan los directores de } \\
\text { todos los ámbitos) y comités } \\
\text { directivos de cada ámbito }\end{array}$ & $\begin{array}{l}\text { Pacientes } \\
\text { crónicos }\end{array}$ & Médico y enfermera \\
\hline 3 & $\begin{array}{l}\text { Apoyo a los clientes internos sobre } \\
\text { cuestiones relacionadas con los } \\
\text { medicamentos } \\
\text { Participación en la discusión de } \\
\text { casos clínicos con el equipo } \\
\text { Integración con las farmacias } \\
\text { comunitarias para discusión de } \\
\text { casos }\end{array}$ & Las pautas de armonización & $\begin{array}{l}\text { Procesos } \\
\text { crónicos, } \\
\text { polimedicados }\end{array}$ & $\begin{array}{l}\text { Médico, enfermero y } \\
\text { farmacéutico }\end{array}$ \\
\hline 4 & $\begin{array}{l}\text { Selección de los medicamentos } \\
\text { Evaluación de los nuevos } \\
\text { medicamentos en las comisiones } \\
\text { de Farmacia y Terapéutica } \\
\text { Evaluación de los medicamentos }\end{array}$ & $\begin{array}{l}\text { Historia clínica compartida } \\
\text { Guía Farmacoterapéutica inter } \\
\text { niveles } \\
\text { Directrices de tratamientos }\end{array}$ & $\begin{array}{l}\text { Procesos } \\
\text { crónicos, } \\
\text { Insuficiencia } \\
\text { Cardíaca, EPOC, } \\
\text { Depresión, } \\
\text { Diabetes, } \\
\text { Insuficiencia } \\
\text { Renal e } \\
\text { Demencias }\end{array}$ & $\begin{array}{l}\text { Médico de familia, } \\
\text { enfermera gestora de } \\
\text { casos y enfermera pre-alta, } \\
\text { farmacéuticos u otros } \\
\text { profesionales de nivel } \\
\text { medio }\end{array}$ \\
\hline 5 & $\begin{array}{l}\text { Selección de los medicamentos } \\
\text { Proporciona evidencia clínica en } \\
\text { la gestión de los casos }\end{array}$ & $\begin{array}{l}\text { Historia clínica compartida } \\
\text { Mensajes por medio de la receta- } \\
\text { electrónica } \\
\text { Indicadores compartidos }\end{array}$ & $\begin{array}{l}\text { Procesos } \\
\text { crónicos, } \\
\text { pacientes } \\
\text { polimedicados }\end{array}$ & $\begin{array}{l}\text { Médico, enfermero, } \\
\text { farmacéutico, auxiliar } \\
\text { técnico de enfermería }\end{array}$ \\
\hline 6 & $\begin{array}{l}\text { Comisión de Farmacia y } \\
\text { Terapéutica inter niveles } \\
\text { Revisión de los protocolos } \\
\text { asistenciales por la farmacia }\end{array}$ & $\begin{array}{l}\text { Comisión de Farmacia y } \\
\text { Terapéutica inter niveles }\end{array}$ & $\begin{array}{l}\text { Procesos crónicos } \\
\text { y dolor }\end{array}$ & $\begin{array}{l}\text { Médico, enfermero, } \\
\text { farmacéutico }\end{array}$ \\
\hline 7 & $\begin{array}{l}\text { Participación en la elaboración de } \\
\text { protocolos asistenciales } \\
\text { Proveer los medicamentos }\end{array}$ & $\begin{array}{l}\text { Comisiones } \\
\text { Protocolos asistenciales y guías } \\
\text { farmacoterapéuticas }\end{array}$ & Procesos agudos & $\begin{array}{l}\text { Médico, farmacéutico, } \\
\text { Enfermera }\end{array}$ \\
\hline 8 & $\begin{array}{l}\text { Selección de los medicamentos } \\
\text { Establecimiento de protocolos } \\
\text { conjuntos con la atención } \\
\text { primaria } \\
\text { Protocolización } \\
\text { farmacoterapéutica }\end{array}$ & $\begin{array}{l}\text { Conciliación de la medicación } \\
\text { Diseño de protocolos } \\
\text { farmacoterapéuticos conjuntos } \\
\text { hospital y atención primaria } \\
\text { Sesiones de trabajo conjuntos } \\
\text { con los diferentes profesionales } \\
\text { Discusiones de casos clínicos }\end{array}$ & $\begin{array}{l}\text { Procesos crónicos } \\
\text { y agudos e } \\
\text { pacientes } \\
\text { polimedicados }\end{array}$ & $\begin{array}{l}\text { Médico, farmacéutico, } \\
\text { otros profesionales de nivel } \\
\text { superior }\end{array}$ \\
\hline
\end{tabular}


ción de informaciones relacionadas a la prescripción, medicación y/o seguimiento farmacoterapéutico. Esta prestación de servicio se realiza a través de la interacción directa con el equipo o a través de la colaboración en la elaboración de guías farmacoterapéuticas y/o la participación en las diferentes comisiones y comités asistenciales. También participan activamente en la selección de medicamentos y en la elaboración de protocolos asistenciales. El grado, profesionalización y formalización de estas actividades es diferente según los entornos. Así mismo, la existencia de elementos y actitudes vinculadas a la coordinación también varía.

Los instrumentos y/o mecanismos de coordinación asistencial verificados en los casos analizados se relacionan con los sistemas de información, el historial clínico compartido, la prescripción y la receta electrónica; guías terapéuticas, protocolos asistenciales, directrices de armonización (pautas); sesiones de trabajos conjuntos y discusión de casos; conciliación de medicamentos; comisiones o comités asistenciales, entre otros. La diferencia entre los casos analizados está en la integración y construcción (o no) conjunta entre los diferentes niveles asistenciales de los instrumentos y mecanismos para la coordinación asistencial.

Los procesos asistenciales priorizados se refieren a los pacientes crónicos y polimedicados, y los profesionales que participan del proceso asistencial son prioritariamente médicos, enfermeros y farmacéuticos.

El Cuadro 4, presenta las herramientas e instrumentos facilitadores implantados en los servicios farmacéuticos para los casos relacionados.

La guía farmacoterapéutica está presente en todos los servicios farmacéuticos, así como las comisiones de farmacia y terapéutica. En relación a los sistemas de información, se observó la presencia

Cuadro 4. Las herramientas y los instrumentos facilitadores de la coordinación farmacoterapéutica, desarrollados por los servicios farmacéuticos, en los diferentes niveles de atención del Sistema Catalán de la Salud. Cataluña, 2016.

\begin{tabular}{|c|c|c|c|c|c|c|c|c|c|}
\hline \multirow[b]{2}{*}{ Casos } & \multicolumn{6}{|c|}{ Lista de Medicamentos } & \multicolumn{3}{|c|}{ Comisión de Farmacia y Terapéutica } \\
\hline & Presencia & \multicolumn{2}{|c|}{$\begin{array}{c}\text { Atiende los } \\
\text { diferentes niveles }\end{array}$} & \multicolumn{3}{|c|}{$\begin{array}{c}\text { Integrada con sistemas } \\
\text { informatizados }\end{array}$} & Presencia & \multicolumn{2}{|c|}{$\begin{array}{c}\text { Composición de profesionales } \\
\text { de diferentes niveles }\end{array}$} \\
\hline \multicolumn{10}{|l|}{1} \\
\hline \multicolumn{10}{|l|}{2} \\
\hline \multicolumn{10}{|l|}{3} \\
\hline \multicolumn{10}{|l|}{4} \\
\hline \multicolumn{10}{|l|}{5} \\
\hline \multicolumn{10}{|l|}{6} \\
\hline \multicolumn{10}{|l|}{7} \\
\hline \multicolumn{10}{|l|}{8} \\
\hline \multirow[b]{2}{*}{ Casos } & \multicolumn{5}{|c|}{ Sistemas de Información } & \multicolumn{4}{|c|}{ Uso Efectivo } \\
\hline & $\begin{array}{r}\text { Histori } \\
\text { Clínica } \\
\text { Informatiz }\end{array}$ & & $\begin{array}{r}\text { Prescripció } \\
\text { integrada } \\
\text { y registro } \\
\text { integrado co } 1 \\
\text { historia clín } \\
\end{array}$ & & $\begin{array}{c}\text { Receta } \\
\text { electrónica } \\
\text { integrada con } \\
\text { la historia } \\
\text { clínica } \\
\end{array}$ & $\begin{array}{r}\text { Mec } \\
\text { ayt } \\
\text { deci } \\
\text { inte } \\
\text { hist }\end{array}$ & $\begin{array}{l}\text { canismos de } \\
\text { uda para la } \\
\text { isión clínica } \\
\text { grados en la } \\
\text { toria clínica } \\
\end{array}$ & \begin{tabular}{|c|} 
Protocolo \\
de manejo \\
o revisión \\
de pacientes \\
polimedicados
\end{tabular} & $\begin{array}{l}\text { Indicadores } \\
\text { de calidad de } \\
\text { prescripción }\end{array}$ \\
\hline \multicolumn{10}{|l|}{1} \\
\hline \multicolumn{10}{|l|}{2} \\
\hline \multicolumn{10}{|l|}{3} \\
\hline \multicolumn{10}{|l|}{4} \\
\hline \multicolumn{10}{|l|}{5} \\
\hline \multicolumn{10}{|l|}{6} \\
\hline \multicolumn{10}{|l|}{7} \\
\hline 8 & & & & & & & & & \\
\hline
\end{tabular}


de historia clínica compartida con su integración a la prescripción electrónica y con receta electrónica en todos los servicios farmacéuticos de las organizaciones sanitarias integradas y en el hospital público, así como en los servicios farmacéuticos de la atención primaria con titularidad pública.

Algunos de los instrumentos implantados en las organizaciones integradas y vinculados a los servicios farmacéuticos para alcanzar el uso efectivo de los medicamentos, se relacionan con los sistemas de información (modelos de revisión concurrente integrados en la historia clínica compartida); con la elaboración de protocolos asistenciales conjuntos dirigidos a los pacientes polimedicados y con el establecimiento de indicadores de calidad de prescripción.

\section{Discusión}

El presente estudio identifica avances que relacionan la percepción y formalización de la coordinación asistencial y clínica de los servicios farmacéuticos asistenciales en el Sistema de Salud de Cataluña. Sin embargo, se identifican también fragilidades y situaciones mejorables en cuanto a la coordinación.

En España, la legislación sanitaria y farmacéutica se ha ido adecuando a los cambios de la profesión. La farmacia queda más dirigida al paciente, incorporando el concepto y las prácticas de los servicios farmacéuticos asistenciales y/o una atención farmacéutica más integrada en el Sistema de Salud. Las leyes de ordenación farmacéutica publicadas por cada Comunidad Autónoma recogen como funciones de los servicios de farmacia de atención primaria, hospitalaria y de las farmacias comunitarias, la colaboración y la actuación coordinada entre ellas ${ }^{11}$.

La Ley de Garantías y Uso Racional de Medicamentos ${ }^{12}$ presenta aspectos esenciales acerca de la profesión farmacéutica, que ha llevado a la implantación de un nuevo modelo de ejercicio profesional que da más relevancia al aspecto asistencial, en el cual el ejercicio profesional del farmacéutico se ha orientado a potenciar la labor como profesional sanitario, sin descuidar las actividades relacionadas con los medicamentos, potenciando la labor del farmacéutico como profesional sanitario.

Parece importante considerar claramente que la Política Farmacéutica y del medicamento debe abordarse de forma integral e integrada con el resto del sistema sanitario y sectores productivos, de forma que permita mejorar los resultados en salud, fomentar el uso racional, seguro, eficaz y eficiente de los medicamentos y promover la gestión eficiente de la prestación farmacéutica, garantizando la sostenibilidad y el acceso a la innovación farmacológica ${ }^{13}$.

Una visión, por tanto, que vaya más allá del coste y visualice el medicamento como una inversión que requiere de la colaboración de todo el sector sanitario, profesionales, gestores, farmacias, distribuidores e industria farmacéutica, en beneficio de las personas y de su calidad de vida ${ }^{13}$.

Los tres tipos de servicios farmacéuticos asistenciales desarrollan sus funciones en distintas organizaciones prestadoras del Sistema de Salud de cada ámbito, y pueden ser responsables de más de un nivel de atención en la misma organización. El objetivo principal de la colaboración entre los servicios farmacéuticos asistenciales de los distintos ámbitos debe ser conseguir una mayor efectividad del tratamiento, prevenir y resolver los posibles problemas relacionados con los medicamentos.

Los profesionales farmacéuticos responsables de los servicios analizados comprenden su misión de acuerdo con las definiciones establecidas en el marco legal del país y de la Comunidad Autónoma de Cataluña ${ }^{14-16}$.

La identificación de los clientes internos y externos en cada servicio farmacéutico asistencial es fundamental para la definición de la cartera de servicios farmacéuticos a ser ofertados en las organizaciones. En las organizaciones sanitarias integradas se observó una cartera de servicios clínicos más ampliada y, posiblemente, más integrada, lo que era previsible debido a la naturaleza de ese tipo de organización.

El Instituto Catalán de Salud (entidad pública proveedora de servicios) ha desarrollado una cartera de servicios farmacéuticos que deben prestar los profesionales en el ámbito de la atención primaria, que contempla siete áreas de procesos dirigidos a la gestión, calidad y seguridad clínica, coordinación entre los niveles asistenciales, formación y docencia, investigación y gestión de medicamentos de uso interno en los centros de salud la cual (la cartera de servicios) pretende armonizar la prestación de esos servicios ${ }^{12}$.

Los servicios farmacéuticos en la Atención Primaria deben aportar, además de los aspectos vinculados al apoyo de los profesionales y usuarios de este nivel asistencial, tres elementos diferenciales: 1) la responsabilidad poblacional, la incorporación del concepto familiar y comunitario en los servicios farmacéuticos y la coordinación con otros ámbitos asistenciales farmacéuticos y no farmacéuticos ${ }^{8}$. 
La perspectiva poblacional es de gran relevancia en la gestión del medicamento, y debe integrarse en la política estratégica y contemplar aquellos aspectos que persiguen optimizar los resultados agregados de la utilización de fármacos, tanto a nivel de efectividad y eficiencia como de seguridad, desde una perspectiva de prevención tanto primaria como secundaria. Es importante diferenciar las actividades dirigidas a optimizar la utilización de medicamentos en pacientes individuales de aquellas dirigidas a garantizar un impacto poblacional ${ }^{8}$.

En Cataluña, se habló por primera vez de la creación y expansión de una cartera de servicios en la farmacia comunitaria en el Plan de Salud 2011-2015, y, en 2012 se alcanzó el primer servicio de cribado de la infección por VIH. En ese ámbito, hubo una progresiva expansión de la indicación de dispositivos personalizados de administración de medicamentos (SPD), que han demostrado su efectividad en el aumento de la adherencia de los tratamientos en pacientes incumplidores ${ }^{17}$.

Además, los servicios farmacéuticos encuadrados en actividades de Promoción de la Salud y Prevención de la Enfermedad, destinados a proveer educación sanitaria o a la prestación de actividades de prevención secundaria, son ejemplos de mayor integración con la comunidad y con el proprio Sistema de Salud.

Como ejemplos tenemos aquellos en el campo de la reducción de daños en usuarios de drogas por vía parenteral, programas de substitución con metadona o de intercambio de jeringuillas, programas de cribado de la infección por VIH poblacional del cáncer colorectal, deshabituación tabáquica, vacunación preventiva, formación sobre uso de medicamentos a personas mayores, o en pacientes con patologías crónicas a fin de promocionar un uso racional y seguro de los medicamentos, o el acompañamiento educativo para el empoderamiento y autogestión de la enfermedad, que se han desarrollado en muchas comunidades autónomas del país.

El estudio para analizar la satisfacción de los participantes en el programa de detección de cáncer colorrectal de Barcelona, evalúan positivamente el programa y apoyan la farmacia como punto de distribución y recolección de tarjetas de prueba de sangre oculta en heces $(\mathrm{FOB})^{18}$. En España, hay una comprensión de las farmacias comunitarias como centro de salud, por su proximidad, accesibilidad, equilibrio territorial, conexión en red y confianza ciudadana, lo que puede complementar idóneamente los progra- mas de prevención primaria y secundaria, como en el caso de la infección por el VIH. No obstante, es cierto que debería asegurarse su correcta derivación, a fin de garantizar su inclusión y seguimiento efectivo en la atención primaria de salud o en las unidades especializadas de referencia ${ }^{19}$.

El estudio realizado en la ciudad de Barcelona para evaluar la experiencia de un trabajo conjunto entre un centro de Atención Primaria y las farmacias comunitarias, apunta una mayor capacidad de actuación, más recursos para solventar problemas, un alto grado de satisfacción de los profesionales y mejores resultados asistenciales para la población ${ }^{20}$.

Otro punto fundamental de la integración del profesional farmacéutico dentro de las rutas asistenciales de los procesos patológicos más prevalentes, en especial de los crónicos, son los programas de conciliación del medicamento en las transiciones asistenciales, en que se aúnan esfuerzos de gestión entre farmacia hospitalaria, farmacia de atención primaria y farmacia comunitaria. Como elemento facilitador para la coordinación entre los servicios farmacéuticos, se observó la implantación de comisiones de farmacia y terapéutica interniveles o territoriales, que parecen ser un instrumento innovador y potente para la coordinación farmacoterapéutica. Estas comisiones buscan elaborar, armonizar y mantener actualizadas las guías farmacoterapéuticas (GFT) que supone una oferta homogénea de medicamentos entre la atención primaria, especializada y socio sanitaria. Están constituidas por un equipo multiprofesional de los diferentes ámbitos asistenciales y clínicos, además de los directivos y disponen de reglamentación específica en cada organización.

La Organización Mundial de la Salud, recomienda que los países implementen comisiones de farmacia y terapéutica en nivel institucional, regional y nacional, una vez que la experiencia ha demostrado los resultados positivos en el control y promoción de la calidad del uso de medicamentos y en la sustentabilidad financiera de las instituciones ${ }^{21,22}$.

Las guías farmacoterapéuticas (GFT) son elaboradas dentro de un proceso de construcción colectivo y bastante largo, en general coordinada por farmacéuticos. Se observó que existe una cultura de incentivo a la participación de los profesionales en esos espacios, con una liberación de carga horaria para la participación en las reuniones y trabajos vinculados a las actividades definidas. Después de la finalización de la guía, se busca una amplia divulgación, ya sea integrándola en los sistemas de información o mediante otras es- 
trategias de difusión. La guía también se utiliza como referencia para la evaluación de la calidad de la prescripción en las organizaciones de salud y supervisión de indicadores establecidos.

Otro instrumento relevante que viabiliza acciones de atención farmacoterapéutica integrada es el historial clínico compartido (HCC) en Cataluña. Esa herramienta, integra documentación clínica relevante del paciente y generada durante todo el proceso de atención en la red de servicios.

Las tecnologías de información y comunicación (TIC) desempeñan un papel fundamental no sólo porque facilitan el acceso a la información clínica relevante de los pacientes, sino también porque permiten nuevas formas de interacción y coordinación entre los diferentes profesionales implicados en la atención (interconsultas virtuales tanto asincrónicas como sincrónicas, consultas virtuales compartidas, envío de imágenes para la interpretación, etc.) y como la comunicación entre usuarios y profesionales (e-consultas, telemonitorización domiciliaria $)^{13}$.

En esta área, Cataluña ha desarrollado la prescripción y la receta electrónica. La prescripción electrónica se presenta como una herramienta estratégica para la coordinación entre los servicios farmacéuticos y la sustentabilidad del sistema, una vez que posibilita la integración de todos los tratamientos existentes para un determinado paciente, independientemente del nivel de cuidado o del establecimiento de salud y del profesional que realizó la prescripción.

Además de eso, facilita la comunicación en los diferentes niveles y una mejor interface de prescripción y dispensación de tratamientos realizados por profesionales diferentes, y se encuentra implantada en todos los centros asistenciales y servicios farmacéuticos de Cataluña.

El estudio realizado por Guayta-Escolies et al. ${ }^{19}$ en hospitales universitarios en Brasil, apunta que la prescripción informatizada representa un gran progreso entre las estrategias utilizadas para minimizar los errores de medicación producidos por prescripciones mal formuladas, sin embargo, no erradica la posibilidad la aparición de error, necesitándose algunas modificaciones ${ }^{23}$.

La receta electrónica, a su vez, posibilita la integración en un único plan de la medicación del paciente y de todas las prescripciones hechas por diferentes profesionales de salud, independientemente del nivel del profesional de salud o entidad, siendo una herramienta esencial para mejorar la calidad de la prescripción y su seguridad clínica ${ }^{24}$.

El proyecto daría respuesta a todo el proceso en que está involucrada la prestación farmacéu- tica, desde la prescripción y la dispensación en la oficina de farmacia, hasta la evaluación y el pago de la prestación ${ }^{24}$. En España, 100\% de las farmacias comunitarias ya están conectadas a la receta electrónica y probablemente es el elemento tecnológico de mayor integración entre los servicios farmacéuticos del Sistema de Salud con las farmacias comunitarias.

Los aumentos apropiados en el uso de la tecnología de la información en la atención de la salud, especialmente la introducción de apoyo a la decisión clínica y mejores vínculos dentro y entre los sistemas, ha resultado en la simplificación del proceso y, además, podrían resultar en una mejora sustancial en la seguridad del paciente ${ }^{25}$, pero, es necesario también atentar sobre la confidencialidad de los pacientes.Se identificaron muchas líneas de mejora en el proceso de coordinación con las farmacias comunitarias, pero apuntamos como principales: la atomización, la no disponibilidad de una población concreta de responsabilidad, la falta de acceso a la historia clínica del paciente, la poca relación con otros profesionales y sobre todo, el sistema de pago del SNS, que se centra única y exclusivamente en pagar el papel logístico de la farmacias comunitarias y la dispensación; cualquier otra actividad de asistencia farmacéutica no está contemplada en la remuneración.

Es imprescindible establecer canales de comunicación formales que faciliten el intercambio de información referente a los medicamentos y también sobre los pacientes con el fin de garantizar una atención farmacéutica integrada, eficiente y de calidad ${ }^{11}$.

En relación a las limitaciones del estudio, el propio diseño del estudio y la muestra, no permite generalizar los resultados para todo el Sistema de Salud de Cataluña, pero permite utilizar los resultados para identificar líneas de mejora y sugerir la formulación de estrategias que puedan incidir en una mayor integración de los servicios farmacéuticos clínicos. Por otro lado, la escasez de estudios con la vertiente cualitativa para el análisis de la contribución de los servicios farmacéuticos en la coordinación asistencial de Sistemas de Salud, dificultó la comparación de los resultados con otras comunidades y/o países.

\section{Conclusión}

La experiencia identificada en el desarrollo de las Políticas Farmacéuticas del Sistema de Salud de Cataluña, con las diferentes herramientas e ins- 
trumentos implantados, parece facilitar una mayor posibilidad de integración entre los servicios farmacéuticos y de éstos con la red de salud.

Las demandas de una sociedad más envejecida, el predominio de enfermedades crónicas, la población más formada en temas relacionados con la salud y el alto consumo de los medicamentos, demanda orientaciones acerca del uso racional de medicamentos a través de servicios farmacéuticos asistenciales de calidad y coordinados para garantizar una farmacoterapia integrada.

En España, y, también en la Comunidad Autónoma de Cataluña, se va imponiendo un nuevo modelo de farmacia, claramente asistencial, con una atención primaria fuerte, aprovechando la infraestructura de la red de farmacias comunitarias, los conocimiento del farmacéutico sobre medicamentos, y las tecnologías de la información y la comunicación.

Así, los servicios farmacéuticos de atención primaria deben jugar un papel central en la gestión de la farmacoterapia integrada y la coordinación con el resto de servicios farmacéuticos, hospitalarios y las farmacias comunitarias, sobre todo en aquellas situaciones de transiciones de los pacientes entre niveles, visando contribuir para una mejor coordinación asistencial y integración clínica en la red de servicios en los Sistemas de Salud.

\section{Colaboradores}

Los autores KS Costa, M Goldbaum y JLS Tolsa participaron da la concepción de la comunicación, análisis e interpretación de los datos, redacción y revisión del contenido. T Guayta-Escolies, $\mathrm{P}$ Modamio y EL Mariño participaron de la revisión del contenido. Todos los autores aprobaron la versión a ser publicada.

\section{Agradecimientos}

Este trabajo se llevó a cabo con el apoyo del Consejo Nacional de Desarrollo Científico y Tecnológico - CNPq del Gobierno de Brasil. Los autores agradecen a los dirigentes de las organizaciones por autorizar la realización de la investigación, y a los entrevistados que aceptaron participar de la misma. Agradecemos al técnico de la Coordinación General de Asistencia Farmacéutica Básica del Ministerio de Salud, Victor Kiiti Tanaka, por su colaboración en el desarrollo del cuestionario electrónico. Agradecemos al Consejo de Colegios Farmacéuticos de Cataluña, la Universidad de Barcelona y la Universidad de São Paulo por su colaboración en la investigación. 


\section{Referencias}

1. Martínez DH, Navarrete MLV, Lorenzo IV. Factores que influyen en la coordinación entre niveles asistenciales según la opinión de directivos y profesionales sanitarios. Gac Sanit 2009; 23(4):280-286.

2. Vázquez ML, Lorenzo IV. Organizaciones sanitarias integradas. Un estudio de casos. Barcelona: Consorci Hospitalari de Catalunya; 2009.

3. Kringos DS, Boerma GW, Hutchinson A, Zee JV, Groenewegen PP. The breadth of primary care: a systematic literature review of its core dimensions. BMC Health Services Research 2010; 10:65.

4. Davis K, Stremikis K, Squires D, Schoen C. Mirror. Mirror on the Wall: How the Performance of the U.S. USA: Health Care System Compares Internationally. New York: The Commonwealth Fund; 2014.

5. Almeida PF, Giovanella L, Mendonca MHM, Escorel S. Desafios à coordenação dos cuidados em saúde: estratégias de integração entre níveis assistenciais em grandes centros urbanos. Cad Saude Publica 2010 26(2):286-298

6. Giovanella L. A atenção primária à saúde nos países da União Européia: configurações e reformas organizacionais na década de 1990. Cad Saude Publica 2006 22(5):951-963.

7. Conill E, Fausto M. Análisis de la problemática de la integración de la APS en el contexto actual: causas que inciden en la fragmentación de servicios y sus efectos en la cohesión social. Rio de Janeiro: EuroSocial Salud; 2007.

8. Ramos AC, Guirado EA, Gorostiaga JM, Juan AMS Tosla LS. Posicionamiento da Sociedade Española de Farmacéuticos en la Atención Primaria en la Gestión Farmacoterpéutica Integrada. Madrid: Editora Ala Oeste; 2015.

9. Organização Panamericana de la Salud (OPAS). Servicios Farmacéuticos basados en la Atención Primaria de Salud: documento de posición de la OPS/OMS. Washington: OPS; 2013

10. Consorci de Salut I Social de Catalunya. Impact on equity of access and efficiency of Integrated Health Care Networks (IHN) in Colombia and Brazil. Equity-LA project id: 223123 (2008-2013). 2013. [artículo da Internet]. [acceso en 2017 ene 05]. Disponible en: http://www2equity-laeu/publications/technical-reports

11. Ramió FC, Lassaletta JC, Jiménez Mc. Integración Farmacéutica. Serveis de Salut Integrats del Baix Empordà 2007. Barcelona: Ediciones Mayo S.A.; 2007.

12. España. Ley de Garantías y Uso Racional de los Medicamentos. [Internet]. [artículo da Internet]. [acceso en 2017 jun 05]. Disponible en: https://www.boe.es/ buscar/doc.php?id=BOE-A-2006-22973

13. Servicio Catalán de Salud. [Internet]. Cataluña. [artículo da Internet]. [acceso en 2016 jul 30]. Disponible en: http://medicaments.gencat.cat/ca/

14. España. Ley 16/2003, de 28 de mayo, de cohesión y calidad del Sistema Nacional de Salud. [Internet]. [acceso en 2016 ago 10]. Disponible en: https://www.boe.es/ buscar/act.php?id=BOE-A-2003-10715
15. Gobierno de España. España. Ley 29/2006, de 26 de julio, de Garantías y Uso Racional de los Medicamentos y Productos Sanitarios. [Internet]. [acceso en 2016 ago 10]. Disponible en: https://www.boe.es/buscar/ pdf/2006/BOE-A-2006-13554-consolidado.pdf

16. Espãna. Ministerio de Sanidad y Consumo. Consenso sobre atención farmacéutica. Madrid. [Internet]. 2001. [acceso en 2016 ago12]. Disponible en: http://www.msssi.gob.es/profesionales/farmacia/consenso/home.htm

17. Informe Farmacia Asistencial. Espãna. [Internet]. [acceso en 2017 ene 25]. Disponible en: www.diariofarma. com

18. Burón A, Posso M, Sivilla J, Grau J, Guayta R, Castells X, Castells A, Macià F, en nombre del Grupo Procolon. Analysis of participant satisfaction in the Barcelona colorectal cancer screening programme: Positive evaluation of the community pharmacy. Gastroenterol Hepatol 2016; 40(4):265-275.

19. Guayta-Escolies R, Giménez A, Rifá B, Barau M, Gascón P, Dronda A, Martínez JL. Cribado de VIH/sida en las farmacias comunitarias. Gac Sanit 2014; 28(3):253259

20. Segú JL. ¿Centros de atención primaria y farmacias comunitarias o un ámbito integrado de atención comunitaria?: la experiencia del barrio de la Barceloneta. Semergen 2004; 30(9):1-5.

21. Laing RO, Hogerzeil HV, Ross-Degnan D. Ten recommendations to improve use of medicines in developing countries. Health Policy Plan 2001; 16(1):13-20.

22. World Health Organization (WHO). WHO medicines strategy: countries at the core. [acceso en 2016 sep 01]. Disponible en: http://whqlibdoc.who.int/hq/2004/ WHO EDM 2012

23. Gimenes FRE, Miasso AI, Lyra Junior DP, Grou CR. Prescripción informatizada como factor contribuyente para la seguridad de los pacientes hospitalizados. Pharmacy Pract (Granada) 2006; 4(1):13-17.

24. Gilabert A, Cubí R. La receta electrónica en Cataluña (Rec@t): ¿prescribimos o recetamos? Aten Primaria 2009; 41(6):298-299.

25. Bates DW, Cohen M, Leape LL, Overhage JM, Shabot MM, Sheridan T. Reducing the frequency of errors in medicine using information technology. J Am Med Inform Assoc 2001; 8(4):299-308.

Artigo apresentado em 26/10/2016

Aprovado em 15/05/2017

Versão final apresentada em 17/05/2017 


\section{Errata}

p. 2595

onde se lê:

Karen Sarmento Costa ${ }^{1}$

Moisés Goldbaum ${ }^{1}$

Rafel Guayta-Escolies ${ }^{1}$

Pilar Modamio ${ }^{1}$

Eduardo Luis Mariño ${ }^{1}$

José Luis Segú Tolsá ${ }^{1}$

leia-se:

Karen Sarmento Costa ${ }^{1}$

Moisés Goldbaum ${ }^{1}$

Rafel Guayta-Escolies ${ }^{2}$

Pilar Modamio ${ }^{3}$

Eduardo Luis Mariño ${ }^{3}$

José Luis Segú Tolsá ${ }^{3}$

onde se lê:

${ }^{1}$ Departamento de Medicina Preventiva, Faculdade de Medicina, Universidade de São Paulo. São Paulo SP Brasil. Av. Dr. Arnaldo 455, Cerqueira César. 01246-903. karen.costa@gmail.com

leia-se:

${ }^{1}$ Departamento de Medicina Preventiva, Faculdade de Medicina, Universidade de São Paulo. Av. Dr. Arnaldo 455, Cerqueira César. 01246-903 São Paulo SP Brasil. karen.costa@gmail.com ${ }^{2}$ Consell de Collegis Farmacèutics de Catalunya. Barcelona Catalunya España.

${ }^{3}$ Unidad de Farmacia Clínica y Farmacoterapia, Departamento de Farmacia y Tecnología Farmacéutica, Facultad de Farmacia y Ciencias de la Alimentación, Universidad de Barcelona. Barcelona Catalunya España. 